\title{
Biotechnology labs reinvented through experiential learning: Enhancing student outcomes through the "flipped lab"
}

\author{
Fei Geng and Faiez Alani \\ School of Engineering Technology, McMaster University, Hamilton, ON \\ gengf@mcmaster.ca
}

\begin{abstract}
This research study involves the use of experiential learning in biotechnology labs to increase student initiative, creativity and problem-solving skills through a "flipped lab" design. A key element for success in undergraduate biotechnology courses is the integration of theory and practice. Biotechnology labs thus require a new synergy of knowledge and experimental implementation. The conventional lab teaching is solely guided by the cookbooklab manual. Although it is important in science for students to learn how to follow directions, offering only cookbook labs limits students' access to exploration. Since the ultimate goal is to allow students to think and behave like engineers, rather than to solely learn or replicate what other engineers have already done, the apparent disadvantage of this conventional teaching method is that it leaves little room for student initiative, creativity and critical problem solving.
\end{abstract}

Biotechnology lab teaching can therefore be greatly enhanced by the application of experiential learning activities. Since experiential learning adapts a student-centered model of education and integrates themes with real-life applicability, biotechnology lab teaching will be more effective if students can play a more active role in lab design and procedures. This paper describes the design of a novel biotechnology lab teaching method: the "flipped lab". A one-year assessment study has been conducted to analyze the effects of the "flipped lab" teaching method on learning outcomes as compared to the conventional model. It quantitatively examines whether the new lab design, with its emphasis on biotechnology-specific technical experience, enhanced students' design lab activities and students' achievement of skills. The results show moderate to significant improvement in all but one of the assessed skills, indicating that the new "flipped lab" design has been largely successful in achieving its goals.

Keywords: Flipped lab; Experiential learning; Biotechnology Lab; The cookbook

\section{INTRODUCTION}

It goes without saying that laboratory training is critical for biotech students by cultivating their technical skills and facilitating the understanding of the key concepts and the related applications. However, the majority of biotechnology labs have been structured in the way that students just need to memorize and follow step-by-step instructions in the lab manuals which are usually prepared by instructors, technicians or publishers. Although it is important in science for students to learn how to follow directions, offering only cookbook labs limits students' access to exploration. Students often do not see the big concept that a cookbook lab is trying to convey.

Students read each step discretely and do not connect the steps to see the bigger intention of the laboratory experience. As the consequence of cookbook lab teaching, students are deficient in the key skills such as lab design, process optimization and troubleshooting.

Cookbook labs do not give the students an authentic sense of the nature of science (Cox and Davis, 1972). Recipe like activities often short-circuit opportunities to stimulate thinking by students (Germann, Haskins, and Auls 1996). A few decades ago educators were wondering why more professors don't provide for open-ended exploration. Then inquiry-based teaching for biotech labs was established in 2005, which provided valuable opportunities and required ongoing intellectual engagement of the students. However, the weakness of this approach is the lack of practice in that students do not have the chance to execute the experiment(s) they are suggesting.

Brownell suggested authentic research-based courses should replace all the Cookbook labs in all the undergraduate biology labs. The research-based lab course had several hallmarks of authentic research: a single longitudinal research focus, research questions with currently unknown answers, student determined experimental designs, and collaboration among lab peers (Brownell et al 2012). However, the lack of supervision in this model and the unpredictable outcomes prevent this new approach from being widely applied.

Due to the apparent disadvantages of the traditional cookbook teaching method, "flipped lab" was developed to allow students to think and behave like engineers rather than to solely learn or replicate what other engineers have already done.

The objective of this project is to improve the biotech lab teaching quality by introducing the novel teaching method "flipped lab". Instead of lab manual, this new method will provide students with a specific simulated scenario (background and research question which needs to be solved). Each student group will be allowed to come up with their 
specific solution to the research question and then to execute it in the real lab setting.

\section{METHODS}

\section{The design of the study}

This study utilized a randomized experiment with student volunteers being randomized into the experimental (flipped lab) and comparison classes (cookbook lab). Prospective students will receive an announcement informing them that they could choose either the existing biotech lab course or volunteer to be randomly selected from a pool that would take the experimental course that would focus on a longitudinal research question. This study used mixed methods including student surveys, laboratory observations, peer evaluation, and student interviews to determine the impact of the two lab conditions on the learning outcome from the students' perspective.

As the most critical component, flipped lab is customized during this project into three stages respectively including fullsupervised, semi-supervised and non-supervised. During each stage, students are going to be provided with a scenario/technical problem together with technical background. Therefore, at each stage students have different amount of flexibility in terms of the selection of lab technology and the creation of experimental procedures. Students are allowed to discuss with their peers to finalize the protocol for the given project. Once the student proposals have been approved by the instructors, the proposed experiments are to be executed during the lab time.

\section{The evaluation of the study}

To evaluate the effectiveness of the flipped lab teaching method, the results including thorough qualitative analysis of interviews and observations, peer evaluation, and the results presenting the pre- and post-course survey data are included. Compared with cookbook teaching method, the major evaluation of flipped lab are Hands-on test and Situational Judgement Test instead of lab reports.

Hands-on Test is a test invigilated by instructors to examine students' laboratory skills and the capacity to conduct the experiment within the limited test period. Based on the learning outcomes from each lab-based course, Hands-on Test can be customized into a variety of forms such as the operation of chromatography equipment to achieve the protein separation. More importantly, Hands-on Test targets individuals rather than groups, which is critical for each lab based course.

Situational Judgement Test is a test invigilated by instructors to examine students' decision making capacity and trouble shooting skills. Each individual is provided with a situation which mimics the real challenges in the industrial environment or academic lab setting. The students need to analyze the situation or the problem and come up with one or multiple technical solutions with the experimental details.

\section{RESULTS AND DISCUSSIONS}

As shown in Fig. 1A, traditional cookbook lab functions in a way that students are spoon-fed with the pre-existing lab manual and instructions from lab professors before their hands-on practice. They only needs to follow the procedure (e.g. microscopy) specified by the manual, collect the data and then write up a lab report for the marking purpose. However, most of the students don't get what the mechanisms are behind the entire lab design. As a matter of fact, they tend to forget the techniques fairly easily due to the sole muscle memory and the lack of the deep understanding.

In comparison, in the Flipped lab (Fig. 1A), instructors need to provide a suitable technical problem for the students one week before the lab time. Student groups then work on the provided technical problem to search for information on the internet and have discussion with the group members to decide the technical plan they are going to pursue. As a prelab assignment, students are required to send a draft proposal to instructors for approval. According to the proposals, lab assistants will prepare lab reagents, chemicals and materials to arrange the lab setup. Based on the amount of details required from the students, flipped lab can categorized into fullsupervised, semi-supervised and non-supervised. Fullsupervised flipped lab model only requires the brief experimental information from the students. Non-supervised flipped lab model requires the complete experimental details.

In this research, Flipped lab was applied to biotechnology students at the level of third year and fourth year as separate groups. We chose ETB-311 biotech lab (Fig. 1B) as the lab location as it is equipped with most advanced multimedia system and data sharing system for all the lab equipment (e.g. fluorescent microscope depicted in the figure). In this lab, students are able to access the lab manual, materials and educational videos through the user portal. All the digital information displayed on the instrument (e.g. microscope) can be duplicated on all the monitors by each lab bench. This type of feature allows students to have real-time discussion on their lab results, which enables them to perform trouble shooting and to modify their lab procedures accordingly.

After the students complete the Flipped lab, they need to complete the peer evaluation form for each member on their team. One week after the lab time, Hands-on Test and Situational Judgement Test are to be hosted to examine their practical skills and project design skills. 
A

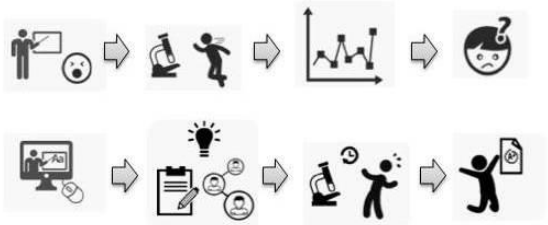

B

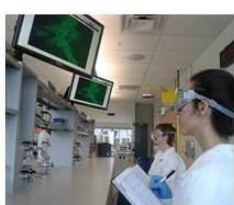

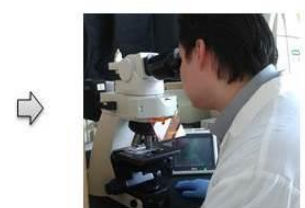

Traditional Lab Teaching

Flipped Lab Teaching

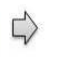

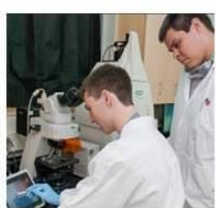

Figure 1. The structural difference between traditional cookbook lab teaching and flipped lab teaching. (A). The demonstration of both traditional lab teaching and flipped lab teaching; (B). The multimedia system in biotechnology lab is critical contributor to the flipped lab teaching.

Compared with the structure of the lab design, the missing of effective marking scheme is another bottleneck which limits the full development of lab based course towards the direction of experiential learning. The marking of the lab course has been quite challenging due to the lack of the corresponding testing system, as well as the difficulty underlying the marking scheme of group work. Thus the most cookbook lab teaching evaluates the learning outcome mostly based on the lab report. As Fig. 2A shows, $90 \%$ of the lab mark has been assignment to lab report in cookbook teaching method. The other percentage has been allocated evenly to pre-lab assignment/quiz or post-lab assignment. As a comparison, flipped lab teaching method combined multiple components into the marking scheme (Fig. 2B). 50\% of the marking measures the student individual lab skills by Handson Test and $15 \%$ of the marking determines the students' project design skills via Situational Judgement Test. $10 \%$ of the lab mark is derived from the peer evaluation and $5 \%$ of the mark is based on the initial proposal.

A

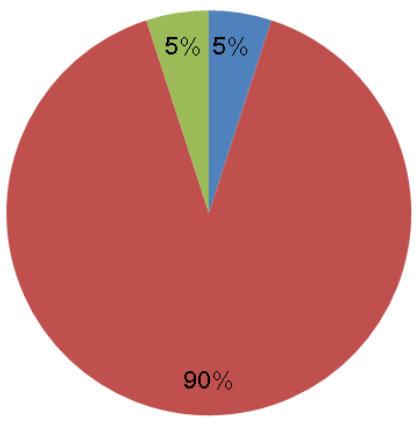

B

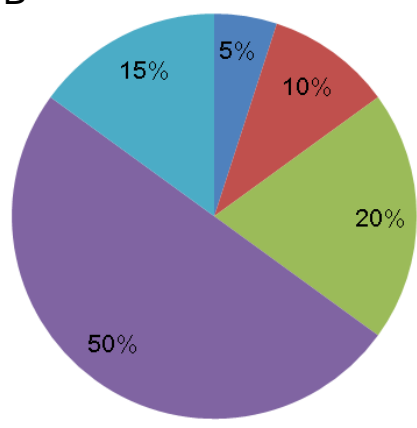

- Pre-lab

- Project participation

Lab report

Hands-on Test

Situational Judgement

Tests

Figure 2. The difference in the marking scheme between traditional cookbook lab teaching (A) and flipped lab teaching (B).

To evaluate the effectiveness of the flipped lab, one of the parameter is student satisfaction. Prior to the start of the flipped lab teaching, the participated students were asked to complete a survey to report their impression about the new teaching method. The survey also asked for their feelings towards common stressors in the biotechnology program, as well as their overall stress level for each year. At the end of the course, students completed the same survey. Both the survey results are compared to reflect the student satisfaction towards the new teaching methods. Fig. 3 shows that students who strongly agreed to recommend the new lab teaching method increased from $70 \%$ to $90 \%$. The results indicated that the flipped teaching method might be more effective in achieving the goals than traditional cookbook teaching method.

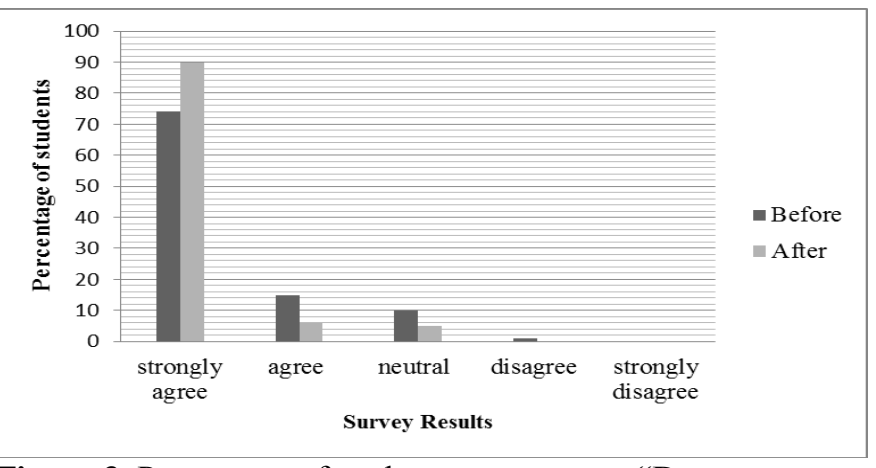

Figure 3. Percentage of students responses to "Do you recommend Flipped lab design for the next year"

- Lab report

Post-lab

Although the student satisfaction has shown a positive sign on this new teaching method, the research progress that has been presented in this paper is still quite preliminary. Much more work need to be done to further improve the new teaching method. Time factor is a major challenge for Flipped lab teaching as the process of proposing ideas, preparation of lab materials accommodating multiple approaches and handson test are all time-consuming. In the future studies, how to 
reach a more sustainable model in the flipped lab design will be the focus.

With the increasing demand from the workplace for students' comprehensive skillset including hands-on skills and project handling skill, laboratory based undergraduate education are facing the major challenges due to the fact that the traditional cookbook teaching method and lab report evaluation system can hardly provide the sufficient training and motivation for students to study. Therefore, flipped lab might be a promising direction together with all the new teaching methods to allow the students to learn rather than to feed them with the information.

\section{ACKNOWLEDGMENT}

This research has been strongly supported by Bachelor of Technology Program at McMaster University. Especially thanks to all the faculty members and technicians in biotechnology stream for the great support.

\section{REFERENCES}

[1] Cox, D.D., and LV. Davis.. The context of biological education: "The case for change". Washington, DC: The American Institute, 1972

[2] Germann P J. 1, Haskins S and Auls S. "Analysis of nine high school biology laboratory manuals: Promoting scientific inquiry" Journal of Research in Science Teaching Vol. 33, No. 5, pp. 475-499, 1996

[3] Brownell S.E., Kloser M.J, Fukami T., and Shavelson R.. "Undergraduate Biology Lab Courses: Comparing the Impact of Traditionally Based "Cookbook" and Authentic Research-Based Courses on Student Lab Experiences," Journal of College Science Teaching, Vol. 41, No. 4, 2012. 